TRANS * núm. $22 \cdot 2018$

ARTÍCULOS · 133-148

En el marco de mi tesis doctoral analicé las traducciones del alemán al euskera de textos de literatura infantil y juvenil y literatura para adultos. Para ello, diseñé y compilé un corpus digitalizado, paralelo y multilingüe. A la hora de diseñar el corpus, se tuvieron en cuenta varios factores y uno de ellos fue el modo de traducción, ya que en algunos casos las traducciones al euskera de textos literarios alemanes se han hecho de forma indirecta a través de una versión intermedia (López Gaseni, 2008; Zubillaga, 2013), en la mayoría de los casos del español. Atendiendo a las características de las traducciones realizadas en la combinación alemán-euskera, los objetivos del presente artículo son mostrar la importancia de realizar un análisis preliminar de catalogación y descripción, explicar el proceso de compilación de corpus y proporcionar los resultados del análisis macrotextual que indican la dificultad de categorizar los textos según el modo de traducción.

PALABRAS CLAVE: estudios descriptivos de traducción, corpus multilingüe, traducción indirecta, euskera, alemán.

\title{
Diseño, descripción y análisis de un corpus multilingüe (alemán-español-euskera)
}

\section{Design, description and analysis of a multilingual corpus (German-Spanish-Basque)}

In the framework of my doctoral thesis, children's and adult literary texts in the language combination German-Basque were analysed. For that purpose, a digitised, parallel and multilingual corpus was designed and compiled. When designing the corpus, different factors - the translation mode, among them - were considered, because in some cases original German literary texts have been translated into Basque through an intermediary version (López Gaseni, 2008; Zubillaga, 2013), the Spanish version in most cases. Taking into

ZURIÑE SANZ VILLAR

Universidad del País Vasco /

Euskal Herriko Unibertsitatea (UPV/EHU) account the features of German-into-Basque translations, the goals of the present paper will be to demonstrate the importance of conducting a preliminary analysis (compilation and description of a catalogue), to describe the creation process of the corpus and to show the results of the macrotextual analysis that indicate the difficulty of classifying the texts according to the mode of translation.

KEY WORDS: descriptive translation studies, multilingual corpus, indirect translation, basque, german 


\section{INTRODUCCIÓN}

La actividad traductora en el País Vasco ha sido un elemento clave en el desarrollo de una lengua minoritaria como el euskera, tanto por su contribución a la producción literaria local, como también por el importante papel que desempeña en el desarrollo y normalización de la lengua. En ese proceso, tal y como indica Uribarri (2005), ante la ausencia de un uso normalizado del euskera en muchos ámbitos, los traductores han adoptado un rol adicional, el de normalizadores de la lengua: «Diese Situation hat den Übersetzern dazu gezwungen, eine zusätzliche Aufgabe als Normalisierer der Sprache zu übernehmen, und sie haben ganz neue Gebiete für die Baskische Sprache eröffnet». ${ }^{\text {. }}$

En cuanto a la producción literaria local, en el segundo apartado se proporcionarán datos específicos sobre el número de traducciones realizadas en la combinación alemán-euskera, pero, en términos generales y según datos de Torrealdai (2013:30), cabe destacar que entre los años 2000 y 2012 la media de textos originales escritos en euskera, por un lado, y de traducciones realizadas a esta lengua, por el otro, se sitúa en un $68 \%$ y un $32 \%$ respectivamente. Los datos indican que la proporción de traducciones, respecto a las obras publicadas originalmente en euskera, es mayor que en Francia y en el resto de España (22\%). Sobre la base de estos porcentajes, Torrealdai (2OI3: 3I) añade que las cifras correspondientes a los textos traducidos en euskera son demasiado altas.

También es importante mencionar el contexto sociolingüístico del País Vasco por su influencia en las traducciones. Los resultados

I «Esta situación ha obligado a los traductores a adoptar un rol adicional como normalizadores de la lengua y ha abierto campos totalmente nuevos para la lengua vasca» (traducción de la autora). de la última encuesta sociolingüística (20I6) realizada en la Comunidad Autónoma Vasca indican que la presencia del euskera ha aumentado en comparación con los resultados de las encuestas previas, tanto en contextos formales como informales. Sin embargo, los datos indican que el $63,3 \%$ de la población mayor de i6 años es monolingüe de castellano, utiliza esta lengua en su vida diaria, y que es el 30,6 \% el que, en mayor o menor medida, hace uso del euskera. Un pequeño porcentaje $(6, \mathrm{I} \%)$ utiliza la lengua minoritaria, pero con muy poca frecuencia. Por lo tanto, a pesar del aumento, sigue habiendo desigualdades, y la realidad sociolingüística se puede describir como de bilingüismo diglósico, donde existe un desequilibrio entre ambas lenguas.

Esta situación influye, tal y como se ha mencionado, en la actividad traductora, y, en el marco académico, también tiene influencia en la metodología que se emplea para analizar las traducciones al euskera. En las traducciones realizadas del alemán al euskera, que serán objeto de estudio en el presente artículo, la gran mayoría de traductores dominan el euskera y el español (y/o el francés). A pesar de estar traduciendo al euskera, el hecho de que en la mente y vida diaria del traductor esté presente la lengua mayoritaria influye en el proceso de traducción (Sanz-Villar 20I8). Además, suele ser habitual que, debido a la debilidad del sistema literario, existan traducciones en otros idiomas de obras que se van a traducir por primera vez del alemán al euskera. La consulta de dichas traducciones previas realizadas a otras lenguas también puede dejar huella en las traducciones vascas. Otro factor decisivo puede ser la limitada disponibilidad de herramientas directas — como, por ejemplo, diccionarios alemán-euskera- que el traductor puede consultar a la hora de realizar las traducciones y el uso, por consiguiente, de 
instrumentos indirectos - es decir, vías indirectas como diccionarios alemán-español y español-euskera-.

En el marco de mi tesis doctoral, compilé un corpus de traducciones realizadas del alemán al euskera con el objetivo de analizar las traducciones en esta combinación de lenguas. Para ello, se siguió el modelo propuesto por Lambert y van Gorp en el año I985, que ya había sido utilizado con éxito en estudios traductológicos anteriores al mío (Merino 1994; Barambones, 2009; Manterola, 2orr o Zubillaga, 2013). Cada investigador puede adaptarlo a sus propias necesidades y el análisis no se limita al estudio de textos descontextualizados, sino que los textos son parte de un sistema. En palabras de Hermans (1999: 65): «[the model] needs to account for a range of other factors in addition to the donor and receptor texts. This is why it is conceived as a multi-level operation, based on communication scheme». En el presente estudio, la realidad de la lengua vasca o la situación sociolingüística han sido factores muy importantes a lo largo de todo el proceso de investigación.

El primer nivel dentro de este modelo consiste en la realización de un análisis preliminar (preliminary data), lo que en mi tesis se traduce, en primer lugar, en la elaboración y descripción de un catálogo —el catálogo Aleuska- o, en otras palabras, el inventario de las traducciones literarias realizadas del alemán al euskera a lo largo de la historia; y en segundo lugar, en la selección de textos y creación del corpus digitalizado, paralelo y multilingüe. Como se reflejará en secciones posteriores, la identificación del modo de traducción (directo, indirecto, etc.) ha sido clave en todos los niveles, empezando desde este nivel preliminar; por ejemplo, ha sido importante de cara a la selección de textos que formarían parte del corpus. Durante la compilación del corpus, por otro lado, se ha incluido la versión intermedia en español, siempre y cuando se haya podido confirmar el carácter indirecto de una traducción.

En un segundo nivel macrotextual (macrolevel), se lleva a cabo el análisis paratextual de los textos que componen el corpus y el análisis de las traducciones de dichos paratextos, entendiendo como paratexto aquellos elementos que se sitúan tanto dentro (títulos, subtítulos, imágenes, etc.) como fuera (críticas, entrevistas, cartas, etc.) del texto (Genette, 1987). Tal y como indica Tahir-Gürçağlar (2002: 45), el análisis microtextual puede ofrecer indicios del estatus de traducción de un texto (por medio de una sintaxis poco habitual, uso de nombres extranjeros, etc.), pero la mayoría de las pistas las encontramos en una serie de elementos, tales como el título, el nombre del autor, el nombre del traductor, el título original, que se sitúan antes del propio texto traducido. Se sitúan alrededor del texto traducido - en contraportada, portada, prólogo, entrevistas a traductores-, en lo que Genette llama "paratexto».

En un tercer y en un cuarto nivel, se realiza el análisis microtextual (micro-level), que aquí consiste en el análisis de la traducción de determinadas unidades fraseológicas, así como un análisis sistémico (systemic context), que engloba todos los niveles. ${ }^{2}$

En este artículo nos centraremos en el análisis preliminar y macrotextual, porque el objetivo es mostrar lo importante que ha sido catalogar las obras traducidas del alemán al euskera a lo largo de la historia, reunir información lo más detallada posible de cada una de ellas y describir el catálogo; un segundo objetivo consistirá en exponer brevemente el proceso de compilación de corpus, donde las versiones puente juegan un

\footnotetext{
2 Los resultados de este análisis se pueden consultar en Sanz-Villar (20I6) y Sanz-Villar (20I8).
} 
papel importante, y, en último lugar, se mostrará la dificultad de categorizar los textos según el modo de traducción por medio del análisis macrotextual.

\section{CATÁlOGO ALEUSKA Y COMPILACIÓN DEL CORPUS}

\section{Corpus o}

Tal y como se ha mencionado, el catálogo Aleuska es un inventario de obras traducidas del alemán al euskera a lo largo de la historia. Para la creación de este catálogo y el consiguiente corpus, nos hemos basado en los pasos propuestos en el seno del proyecto de investigación TRACE. ${ }^{3}$ En palabras de Gutiérrez Lanza (2007), miembro del grupo: «En el marco del proyecto TRACE un Corpus o/Catálogo es un listado lo más exhaustivo posible, preferentemente informatizado, en cuyo caso adquiere categoría de "base de datos" (Rabadán y Merino 2004), de fichas que contienen diversos campos de información (con)textual (...)».

Fue el profesor de la UPV/EHU Ibon Uribarri el que, en la combinación alemán-euskera, comenzó esta labor de catalogación en el año 2003, y la profesora Naroa Zubillaga, de la misma universidad, y yo hemos continuado con esa tarea en el marco de nuestras respectivas tesis doctorales. Para la creación del catálogo Aleuska, se utilizó el programa Filemaker, donde por cada obra identificada se intenta recopilar la siguiente información: título origen y título meta, autor origen y autor meta, fecha de publicación del texto original (TO) y del texto meta (TM), editorial del TM, lugar de publicación del TM, número de páginas del

3 Se trata de un proyecto de investigación sobre traducciones censuradas. En la página web del proyecto (http://www.ehu.es/trace/colectivo.html) se puede obtener información más detallada.
TM, género y etiqueta meta. La identificación o selección de las obras se realizó mediante búsquedas en bases de datos, tales como Index Translationum, y en los catálogos de diversas bibliotecas (el catálogo online de bibliotecas de Euskadi así como el de la biblioteca Azkue, biblioteca Sancho el Sabio, biblioteca Koldo Mitxelena, la Biblioteca Nacional de España o la Deutsche Nationalbibliothek).

En términos generales, no fue difícil recopilar los datos mencionados y seguir actualizando, de esta forma, el catálogo. El mayor obstáculo fue identificar el modo de traducción en esta fase de catalogación -es decir, si los textos habían sido traducidos directamente del original alemán o si se había utilizado alguna versión intermedia para llevar a cabo la traducción-, ya que las bases de datos y los catálogos mencionados en líneas anteriores no suelen recoger dicha información. Por esta razón, en caso de duda nos pusimos en contacto con editoriales y traductores. Cuando no obtuvimos respuesta o no pudimos establecer el contacto, introdujimos un signo de interrogación en el campo «modo de traducción» del catálogo. En cualquier caso, no hay que olvidar que podemos actualizar la información del catálogo según los resultados de los análisis macro y microtextuales.

El catálogo, que de momento está actualizado hasta noviembre del 20I3, cuenta con un total de 7 Io entradas, incluyendo las reediciones. Si clasificamos los textos por tipo textual, obtenemos la distribución que se presenta en la figura $\mathrm{I}$.

Tal y como se puede observar en el grafico I, los textos de literatura infantil y juvenil (LIJ) son el tipo de texto que más se ha importado (445 libros). En segunda posición, se sitúa la poesía con I44 entradas, aunque es necesario mencionar que muchas de las fichas del catálogo correspondientes a este género están 


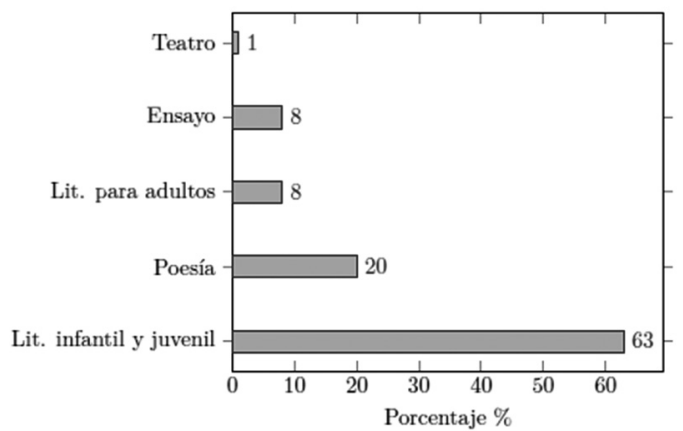

Figura I. Distribución de tipos textuales en el catálogo Aleuska

formadas por un único poema. Después, con representación similar, nos encontramos con textos de literatura para adultos (LA, 59 obras) y ensayos (54), y por último, cabe mencionar que sólo contamos con 8 obras de teatro.

Escogimos textos tanto de LA como de LIJ para la compilación del corpus por cuestiones de representatividad en el catálogo y porque nos interesaba realizar la comparación entre la traducción de estos dos tipos de texto. Antes de comenzar con el proceso de creación del corpus, fue necesario realizar una descripción exhaustiva del subcatálogo LA-LIJ para obtener información clara sobre los autores originales, el desarrollo de las publicaciones de los TM, los traductores y el modo de traducción.

En relación con los autores originales, los datos del subcatálogo muestran que se han traducido al euskera obras de 126 autores alemanes diferentes, 96 en LIJ y 32 en LA. Teniendo en cuenta el número de textos correspondientes a LIJ y LA (284 y 48 respectivamente, sin tener en cuenta las reediciones), podemos concluir que la diversidad de autores originales es mayor en LA $o$, en otras palabras, que de media se han traducido más obras del mismo autor alemán en LIJ.

$\mathrm{Al}$ analizar la evolución de las traducciones vascas, se puede observar una línea clara antes y después del año 1980: mientras que antes de esta fecha la tendencia era traducir una obra por año, la situación cambia por completo a partir de I980, cuando se empieza a publicar una media de 9 libros por año (sin tener en cuenta las reediciones, I4 si las contamos). Es importante tener en cuenta el contexto político de la época para analizar dicho cambio; por ejemplo, fue muy importante la entrada en vigor de la Ley Básica de Normalización del Uso del Euskera en el año 1982, hecho que favoreció el uso del euskera y la producción de material en esta lengua. A partir de este momento se inicia una actividad continua en el sector de la traducción al euskera (Uribarri, 2005).

Resulta interesante distinguir entre LA y LIJ por las diferencias tan marcadas que existen entre el desarrollo de uno y otro género, y que se pueden apreciar en los gráficos 2 y 3 .

La línea gris oscura indica el número de traducciones de LA teniendo en cuenta las reediciones. Apenas se aprecia en el gráfico 2, debido a que se han contabilizado muy pocas reediciones de traducciones de LA. Además, la evolución ha sido muy regular, a excepción de un pequeño pico en el año I992 cuando se tradujeron seis obras del alemán. Cabe destacar que cuatro de ellas se publicaron en el marco de la colección Literatura Unibertsala, iniciativa creada en 1989 gracias a un convenio de colaboración entre EIZIE ${ }^{4}$ y el Gobierno Vasco, con el fin de «poner al alcance de los lectores vascoparlantes un amplio abanico de obras representativas de la literatura universal traducidas al euskera». ${ }^{5}$ En el desarrollo de las traducciones de LIJ, por otro lado, encontramos

4 La Asociación de Traductores, Correctores e Intérpretes de Lengua Vasca.

$5 \mathrm{http} / /$ www.eizie.eus/es/Argitalpenak/

Literatura_Unibertsala. 


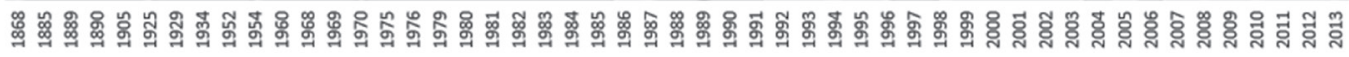

Figura 2. Traducciones de LA publicadas entre 1868 y 2013

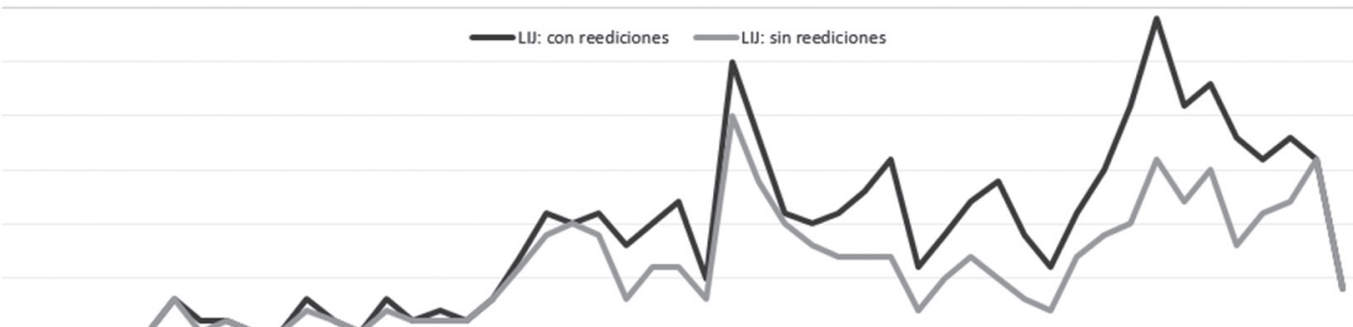

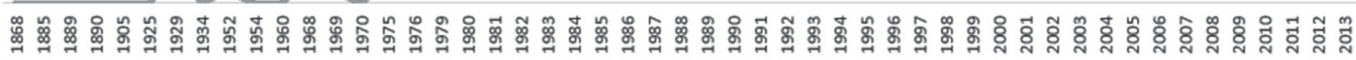

Figura 3. Traducciones de LIJ publicadas entre I868 y 2013

muchas más reediciones y más altibajos (figura 3). Hay dos años que destacan por el alto número de publicaciones: 1990 y 2006. En el primero, Io de las 25 publicaciones pertenecen a la misma colección de historias cortas escritas por Sabine Lohf y traducidas por Joxantonio Ormazabal. En el 2006, el pico se debe a las I3 reediciones de Io traducciones de libros originales escritos por el autor alemán Knister, dentro de la serie Hexe Lilli (Kika Superbruja).

Han sido 127 los traductores que han traducido las obras recogidas en el subcatálogo de LA y LIJ. Teniendo en cuenta el número de obras traducidas, se puede concluir que hay muy pocos traductores que se han especializado en la traducción alemán-euskera, ya que es una gran mayoría la que ha traducido una única obra en esta combinación lingüística. En el polo opuesto, Pello Zabaleta y Xabier Mendiguren son los más prolíficos con 33 y 29 traducciones respectivamente; además, ambos han traducido obras tanto en LA como en LIJ.
El último aspecto que se ha descrito en el subcatálogo Aleuska ha sido el modo de traducción de las obras de LA y LIJ, como se ha mencionado en líneas anteriores. Debido a la dificultad de obtener esta información, los textos que se han identificado como directos o indirectos a este nivel hay que considerarlos como supuestas traducciones directas e indirectas - en el sentido de assumed translation de Toury (2012), pero ampliado al modo de traducción en este caso- - Los resultados de los posteriores análisis a niveles macro y microtextual servirán para actualizar la información recogida a nivel de catálogo o Corpus o. A nivel conceptual, entendemos como traducción indirecta la que se realiza por medio de una versión intermedia, y no de la versión original alemana (en este caso), mientras que una traducción directa es la que se ha realizado principalmente de la versión original alemana, es decir, el traductor puede haber consultado otras versiones, pero la versión principal es la alemana. 


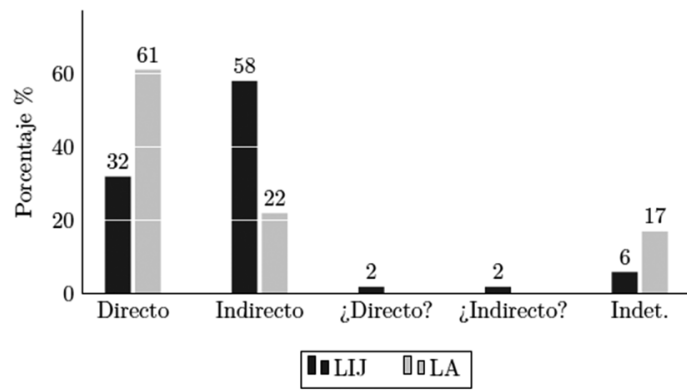

Figura 4. El modo de traducción en las traducciones de LIJ y $L A$

Tal y como se puede observar en la figura 4, en las traducciones de LIJ la norma la constituyen las supuestas traducciones indirectas (58\%) $\mathrm{y}$, sin embargo, en LA lo que predominan son las supuestas traducciones directas (6r \%). Como se ha indicado anteriormente, hay una serie de obras que no han podido ser clasificadas o se han clasificado con signos de interrogación por falta de información.

El número de traducciones realizadas por un traductor y el modo de traducción podrían estar relacionados. Zubillaga (2013: 447) lo analiza en su tesis en referencia a las traducciones de textos de LIJ del alemán al euskera, pero concluye que ella no ha podido confirmar la hipótesis - es decir, que traducir un número considerado de obras no necesariamente tiene que significar que se traduzca de forma directa- y que hay que considerar otros factores. Sin embargo, en los textos de LA, puede haber cierta relación, ya que las traducciones indirectas se dan, sobre todo, entre los traductores que han traducido una única obra. Por otro lado, no podemos olvidar que puede haber otros factores, tales como el prestigio del género textual, que también pueden haber influido en estos resultados.

\section{Corpus I}

En un paso intermedio entre la creación y descripción del catálogo, por un lado, y el análisis macrotextual, por el otro, es imprescindible definir, teniendo en cuenta los resultados obtenidos del análisis del catálogo Aleuska, los criterios para la selección de los textos que formarán el corpus; o en otras palabras, será necesario crear el corpus I. Como indica Merino (2003: 650): «(...) la noción de corpus I o primario (y su correspondiente de corpus secundario) ha resultado indispensable como recurso metodológico en estudios descriptivos acometidos desde muy diferentes perspectivas».

En primer lugar, se han seleccionado textos traducidos a partir de 1980 , momento a partir del cual se da un crecimiento considerable en el número de traducciones al euskera. En segundo lugar, de los diferentes tipos de texto que tienen representación en el catálogo, se han elegido textos del subcatálogo LA-LIJ, principalmente porque estos textos representan el 7I \% del catálogo. El tercer criterio está relacionado con el modo de traducción. Como acabamos de mencionar, se han identificado tanto traducciones directas como indirectas en el subcatálogo. En el presente corpus, ambos tipos de traducciones tendrán representación, aunque habrá más supuestas traducciones directas (alrededor del $70 \%$ del corpus frente al $30 \%$ de traducciones indirectas), porque nuestro interés principal radica en los resultados de las primeras. Por último, como el objetivo no es analizar las traducciones de un único autor original o un único traductor, la diversidad de autores será un factor adicional a tener en cuenta.

En resumidas cuentas, el corpus está compuesto por 24 textos de LIJ y 24 textos de LA. En las traducciones de LIJ, I5 son supuestas traducciones directas y 9 indirectas. Como el número de traducciones indirectas no es tan elevado en LA, 
se han escogido I9 supuestas traducciones directas y 5 traducciones indirectas. En cuanto a la diversidad de autores, el corpus contará con obras 140 de 30 autores originales diferentes y 28 traductores diferentes. En la siguiente tabla, se puede ver un resumen de las características del corpus I:

Tabla 1. Características del corpus 1

\begin{tabular}{lllll}
\hline \multicolumn{4}{c}{ Modo de traducción } & Autores \\
\hline Directo & Indirecto & $\begin{array}{l}\text { Autor } \\
\text { original }\end{array}$ & $\begin{array}{l}\text { Autor } \\
\text { meta }\end{array}$ \\
\hline LA & I9 & 5 & I7 & I5 \\
\hline LIJ & I5 & 9 & I3 & I6 \\
\hline Total & 34 & I4 & 30 & 3I \\
\hline
\end{tabular}

En las I4 traducciones indirectas, no solo se han incluido en el corpus el original alemán y la traducción al euskera, sino que ha sido de especial relevancia para los posteriores análisis añadir la versión intermedia. De esta forma, hemos creado un corpus digitalizado, paralelo y multilingüe (alemán, español, euskera). De cara a la propia compilación del corpus, son cuatro los pasos principales que se han seguido: digitalizar, limpiar, etiquetar y alinear.

Como es bien sabido, digitalizar los textos es un trabajo costoso. Algunas editoriales nos proporcionaron los textos en formato digital, gracias a lo cual pudimos prescindir de este paso en ciertas ocasiones. Para limpiar, etiquetar y alinear los textos utilizamos un programa creado primero en el marco del proyecto TRACE y desarrollado más tarde por el informático Iñaki Albisua dentro del grupo de investigación TRALIMA-ITZULIK de la UPV/EHU: el programa TAligner.

Se trata de un programa escrito en Java, muy intuitivo, que permite realizar la limpieza de los textos en cuestión de pocos segundos, el etique- tado de los textos a nivel de párrafo y de oración (adjudicando etiquetas para numerar cada oración y párrafo), y la alineación simultánea de múltiples textos. Esta fue una de las razones por las que se decidió crear una herramienta propia para la compilación de corpus. Si queríamos analizar traducciones al euskera, era imprescindible trabajar con una herramienta que fuera capaz de alinear más de dos textos al mismo tiempo. La alineación no es automática, el usuario tiene que ajustar de forma manual los segmentos para obtener un texto bien alineado; es decir, para que cada segmento original corresponda a cada segmento meta o a los segmentos meta, es preciso realizar ajustes manuales. Aunque es un proceso largo, de esta forma el investigador tiene la seguridad de que la alineación es correcta, y los problemas de alineación se reducen al mínimo cuando se realizan búsquedas en el corpus.

Las búsquedas se pueden realizar en el mismo TAligner, haciendo clic en la pestaña «Consultar corpus» que se puede ver en la figura 5.

Gracias a los metadatos que hemos introducido para cada texto en el proceso de etiquetado, podemos limitar las búsquedas atendiendo a diversos factores: autores, traductores, idioma, modo de traducción, etc. En los resultados de las búsquedas se muestra la oración que contiene la palabra (o palabras) que se ha(n) buscado, la oración anterior y posterior (esta ampliación del contexto es opcional) y las correspondientes traducciones. En la figura I, como el texto ED (Emil und die Detektive) se ha traducido de forma indirecta, nos aparecen los correspondientes fragmentos en español y en euskera.

\section{ANÁLISIS MACROTEXTUAL}

Una vez seleccionados los textos que han pasado a formar parte del corpus, el siguiente paso, según la metodología expuesta en la introduc- 


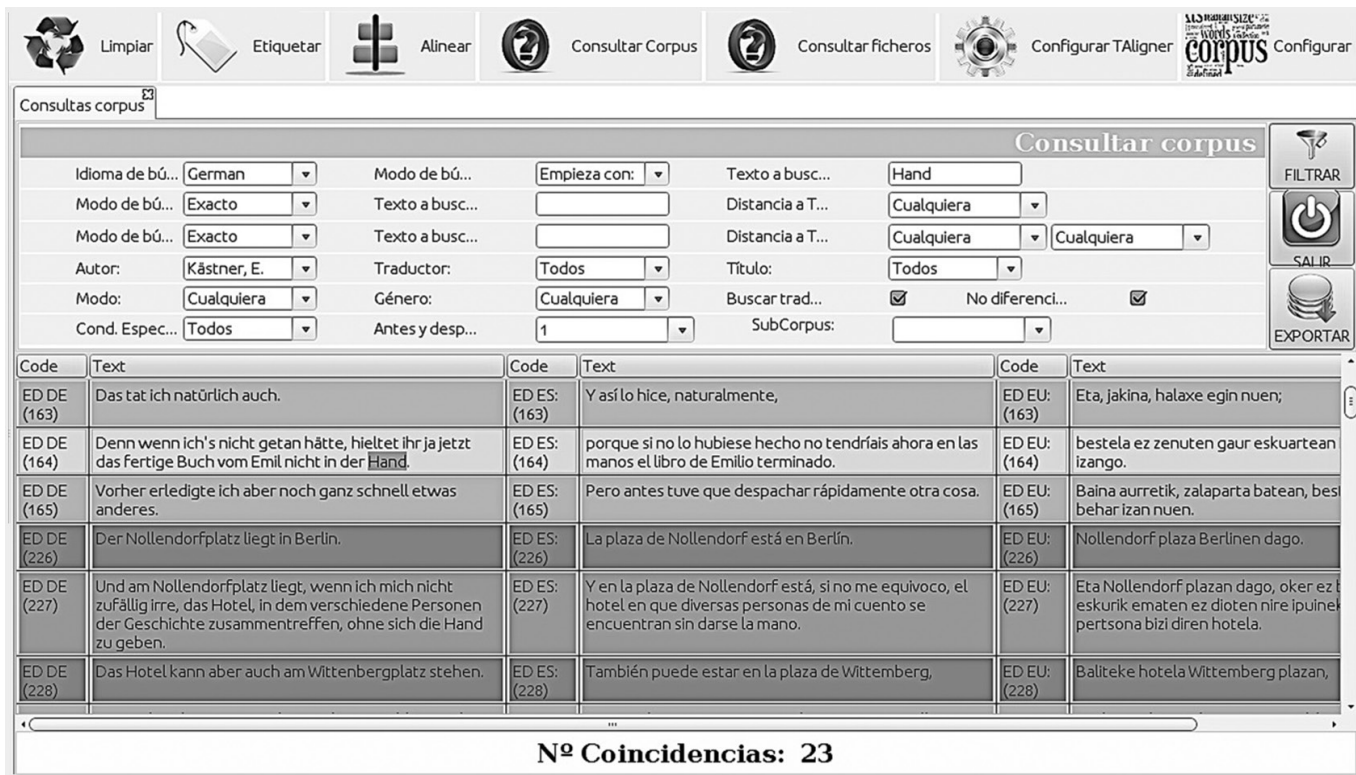

Figura 5. Búsquedas en el programa TAligner

ción de este artículo, consiste en la realización de un análisis macrotextual de dichos textos, lo que se traduce en el análisis de los paratextos y la traducción de determinados elementos paratextuales. Según Genette (1987), los paratextos están formados por peritextos - aquellos elementos, tales como, título, subtítulo, prólogo, epílogo, notas, etc., que se sitúan dentro de la obra-y por epitextos - es decir, elementos que se sitúan fuera del texto, como críticas, entrevistas, correspondencia privada, etc. - (Garrido Vilariño, 2orr: 69-79). En nuestro caso, mediante el análisis paratextual será relevante analizar la localización de los nombres de los autores originales, la visibilidad del proceso de traducción así como la información que nos proporcionan traductores, editoriales y otras fuentes epitextuales.

\section{Análisis paratextual}

En los 48 textos meta en euskera que forman parte del corpus, en todos y cada uno de ellos aparece el nombre del autor original alemán y el del traductor. Además, mientras que el primero, el nombre del autor alemán, se muestra siempre en la portada de los libros, como es habitual, el nombre del traductor solo se puede observar en la portada en I8 ocasiones y todos ellos corresponden a títulos de LA. Por lo tanto, se puede concluir que en LA la visibilidad del traductor es mayor que en LIJ, y que dentro de los textos de LA, en la mayoría de los casos (75\%) el nombre del traductor aparece en la portada. Tanto en LA como en LIJ, parece que hay relación entre el posicionamiento de estos elementos paratextuales (el nombre del autor y del traductor) y las editoriales, ya que dentro de la misma editorial se han observado las mismas tendencias. Encontramos una excepción: entre los textos de LA en los que el nombre del traductor no aparece en portada, dos pertenecen a la misma editorial y, sin embargo, en posteriores publicaciones de esa misma editorial, sí que se 
observan los nombres de los traductores en la portada. Por lo tanto, en este caso concreto la política de la editorial en cuanto a la visibilidad del traductor ha cambiado a lo largo del tiempo.

En referencia a la visibilidad del proceso de traducción, hay ciertos elementos paratextuales que nos pueden permitir descubrir en qué medida los paratextos muestran cuál es el texto de partida y, en las traducciones indirectas, cuál es o cuáles son las versiones intermedias utilizadas. Estos elementos son la explicitación tanto del título original como de la lengua original y la presencia de información relacionada con alguna versión intermedia.

La tendencia en cuanto a la aparición del título original alemán en los paratextos es la de incluirlo, ya que esto ocurre en 42 de los 48 textos del corpus. Si nos fijamos en lo que ocurre en las traducciones que hemos identificado como indirectas, veremos que la mayoría de las veces (II de I4), el título original alemán aparece en alguna parte del libro. En este sentido, si se argumenta que la inclusión del título original en los paratextos puede indicar un intento de esconder el carácter indirecto de la traducción, podemos decir que existe dicha tendencia en el presente corpus. No ocurre lo mismo con la explicitación de la lengua original, ya que solo en cuatro casos se indica de forma explícita que la traducción se ha realizado del alemán. Como dato curioso, tres de ellos han sido traducidos por el mismo traductor. Sería interesante analizar en qué medida el traductor ha podido influir en la aparición de esta información paratextual.

El hecho de que aparezca información sobre algún texto intermedio en los paratextos puede apuntar hacia el carácter indirecto de dicha traducción. En el corpus, se han encontrado solo cinco libros donde aparece información explícita sobre una versión intermedia en español; todos ellos pertenecen a textos de LIJ y la mayo- ría han sido publicados por editoriales españolas (o alianzas de editoriales españolas y vascas).

Ruzicka (2002: 8-9) identifica tres factores por los cuales ciertas editoriales con larga tradición en publicar textos de LIJ en español se interesaron a partir de los años 70 y 80 por los mercados de aquellas comunidades autónomas donde coexistían dos idiomas:

- Los gobiernos de esas comunidades autónomas empezaron a financiar de forma directa (mediante ayudas económicas) o indirecta (mediante la compra de ejemplares de dichas traducciones) las traducciones a las lenguas autóctonas.

- Las traducciones al euskera a partir de versiones españolas eran atractivas desde el punto de vista económico, ya que no eran muy caras.

- La situación legal en cuanto a la compra de derechos de traducción era propicia. En la mayoría de los casos, si una editorial española compraba los derechos para traducir una obra, el contrato incluía una cláusula que permitía a la editorial realizar traducciones de la misma obra a todas las lenguas peninsulares.

En cuanto a la información obtenida por traductores y editoriales, cabe mencionar que ha sido muy valioso tener datos de primera mano sobre los textos que han utilizado los traductores en el proceso traductor. En dos traducciones directas, las traductoras nos comunicaron, por ejemplo, que la versión original de la que habían partido había sido la alemana, pero que también habían consultado otras versiones en caso de duda. En otra traducción indirecta, la traductora utilizó tres versiones diferentes (la inglesa, la francesa y la española) para realizar dicha traducción, y no es el único ejemplo de traducción indirecta en el que se ha hecho 
uso de diferentes versiones. También ha sido importante la información recibida por parte de las editoriales, aunque como apunta Zubillaga (2013: 4I2), el hecho de que la editorial proporcione al traductor la versión original alemana no necesariamente quiere decir que hayan utilizado ese texto como texto principal durante la traducción. Otra fuente de información han sido las entrevistas realizadas a algunos traductores y publicadas en la revista Senez. ${ }^{6}$ Dos de ellos dejan claro que realizan las traducciones desde la versión original alemana y Zabaleta, además, hace referencia a la necesidad de usar herramientas indirectas (diccionarios alemánespañol) cuando se traduce desde el alemán al euskera (Biguri y Zabaleta, 1990).

\section{Análisis de la traducción de elementos paratextuales}

Garrido Vilariño indica que «if translation is the reading, interpreting and transfer of texts, then paratranslation is the reading, interpreting and transfer of paratexts» (2orr: 76), término propuesto por el grupo de investigación Traducción \& Paratraducción de la Universidad de Vigo de la que el investigador forma parte. Distingue entre tres tipos de paratextos: verbales, icónicos y verbo-icónicos, y en las siguientes líneas nos ocuparemos de analizar la traducción de los dos primeros. Para ello, nos fijaremos en la traducción de títulos de libros y de nombres de capítulos, y a nivel icónico, compararemos algunas portadas de las obras del corpus.

El trasvase del título de un libro de un idioma a otro va más allá de la traducción (Garrido Vilariño, 20Ir: 73); se trata más de adaptar que de traducir. Dicha traducción/adaptación tendrá

\footnotetext{
6 Revista de periodicidad anual que reúne artículos sobre temas relacionados con la traducción del y al euskera nacida en el seno de la Asociación de Traductores, Correctores e Intérpretes de Lengua Vasca (EIZIE).
}

en cuenta la ideología de la sociedad y del mercado cultural meta, y normalmente no suelen ser los traductores los responsables de dichas traducciones, sino otros mediadores. Sin embargo, «currently, in addition to the various loyalties and the recognized (in)visibilities, translators are generally held responsible for all the good and bad decisions (and rather more often the latter) concerning the success or failure of a text, because it is their names that appear on the covers, or at least on the title pages» (20II: 68).

No sabemos quién es el responsable de la traducción de los títulos de los libros en el presente corpus, pero sí que podemos identificar algunas tendencias. En comparación con los textos de LA, en las traducciones de los títulos de LIJ, en general, es más común alejarse del TO. Es interesante observar, por ejemplo, que en cuatro traducciones directas de LIJ (tabla 2), los títulos se parecen más a las versiones en español que al título original alemán:

Como se puede observar, las versiones en español y euskera comparten palabras que no están presentes en los títulos originales alemanes: Jojo, inglés, locos y mejor defensa.

Otro ejemplo interesante de LIJ es la traducción de un libro de la colección de Hexe Lilli. El nombre de la bruja se traduce al español y al euskera mediante Kika («Kika suberbruja» $\mathrm{y}$ «Kika supersorgina»). No ocurre lo mismo en la traducción a otros idiomas: en italiano se llama Martina, Tina en catalán y se utilizan adaptaciones de Lilli en muchos otros idiomas (Fischer, 20II). Es decir, algunos mantienen el nombre original adaptado o sin adaptar (Lili, Lilly, Lilli) y otros, los libros en italiano, catalán, español y euskera (este último por influencia del español, ya que se trata de una traducción indirecta), utilizan nombres diferentes.

En LA, como se ha comentado, se suele seguir más de cerca al título original alemán. 
Tabla 2: Título originales en alemán y sus traducciones al español y al euskera

\begin{tabular}{lll}
\hline TO & Versión intermedia & TM \\
\hline $\begin{array}{l}\text { Das Gauklermärchen } \\
\text { (Ende, I982) }\end{array}$ & $\begin{array}{l}\text { Jojo, historia de un saltimbanqui } \\
\text { (Ende, I986) }\end{array}$ & $\begin{array}{l}\text { Jojo, pailazo baten historia [Jojo, historia de } \\
\text { un payaso] (Ende, 1995) }\end{array}$ \\
\hline $\begin{array}{l}\text { Das Austauschkind } \\
\text { (Nöstlinger, I982) }\end{array}$ & $\begin{array}{l}\text { Intercambio con un inglés } \\
\text { (Nöstlinger, I986) }\end{array}$ & $\begin{array}{l}\text { Ingeles bat etxean } \text { [Un inglés en casa] } \\
\text { (Nöstlinger, I99I) }\end{array}$ \\
\hline $\begin{array}{l}\text { Bei uns in Schilda } \\
\text { (Preußler, I958) }\end{array}$ & $\begin{array}{l}\text { Los locos de Villasimplona } \\
\text { (Preußler, I988) }\end{array}$ & $\begin{array}{l}\text { Markako eroak [Los locos de Marka] } \\
\text { (Preußler, I987) }\end{array}$ \\
\hline $\begin{array}{l}\text { Juli, die Viererkette } \\
\text { (Massanek, 2003) }\end{array}$ & $\begin{array}{l}\text { Juli, el mejor defensa } \\
\text { (Massanek, 2005) }\end{array}$ & $\begin{array}{l}\text { Juli, defentsa onena [Juli, el/la mejor defensa] } \\
\text { (Massanek, 2oIo) }\end{array}$ \\
\hline
\end{tabular}

Tabla 3: Traducción al español y al euskera del capítulo de un libro

\begin{tabular}{lll}
\hline To & Versión intermedia & TM \\
\hline $\begin{array}{l}\text { Schulwege und Gutenachtküsse } \\
\text { (Kästner, 1949) }\end{array}$ & Cariñosas despedidas (Kästner, 1957) & Agur maitakorrak (Kästner, I992) \\
\hline
\end{tabular}

Hay dos que se alejan un poco más del original $\mathrm{y}$ son precisamente traducciones indirectas.

En el análisis de la traducción de los capítulos, también se han observado algunos indicios que podrían apuntar hacia el uso de otras versiones en traducciones directas. Por ejemplo, en un texto de LIJ (Brezina, I995), uno de los capítulos en alemán se titula "Gibt sie niemals auf?» (¿Nunca se rinde?). Tanto en la versión en español como en la traducción al euskera los traductores han decidido sustituir el pronombre «sie» (ella) por «esta mujer» en español y por «emakume hau» (esta mujer) en euskera: «Es que esta mujer nunca se rinde?», «Emakume honek ez al du etsitzen?».

Encontramos otro ejemplo en una traducción directa de LIJ. En este libro (Kästner, I949), en algunas partes del texto la versión en euskera se aleja bastante del original alemán, y a veces hemos podido comprobar que ha podido ser por influencia de la versión en español, pero otras, sin embargo, no. Veamos un ejemplo en el que la versión intermedia ha podido influir en la traducción meta (tabla 3).

La traducción literal del texto alemán sería «los caminos a la escuela y besos de buenas noches», lo que en español se traduce por cariñosas despedidas, y en euskera, agur maitakorrak, es una traducción directa de la versión española.

Para concluir con este apartado, nos fijaremos en las portadas de los libros. En palabras de Gerber (2008) resulta difícil comparar la «traducción» de las portadas de TO y TM por el hecho de que muchas veces nos encontramos con numerosas portadas diferentes que pertenecen a un mismo texto: «(...) titles (in majority of cases) remain unaltered with the publication of new editions while, in contrast, other peritextual features (including the cover art, illustrations and blurb text) are commonly changed with every new edition» (2008: 42).

Se pueden observar diferentes tendencias en los textos de nuestro corpus. En los textos de LA, prevalece la tendencia de no mantener la 

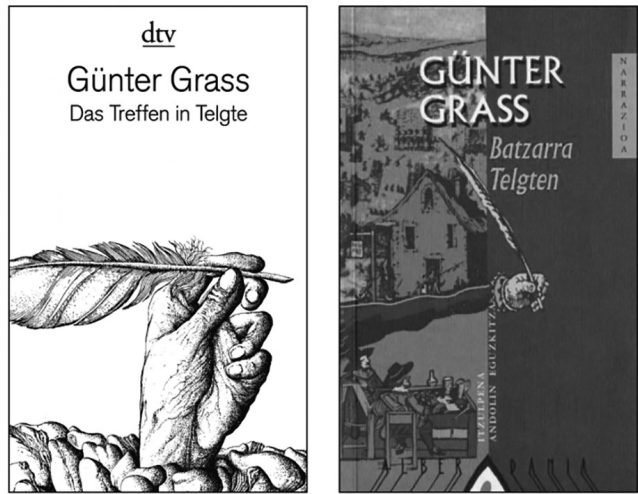

Figura 6. Portadas de Das Treffen in Telgte (Grass, I979) y Biltzarra Telgten (Grass, 2002)
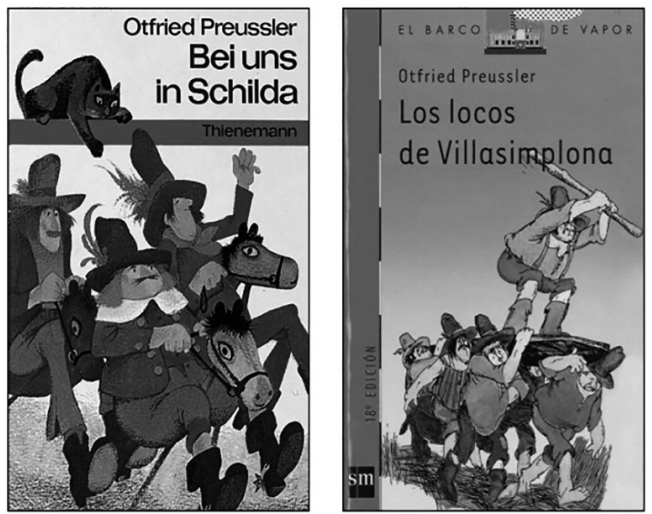

portada del texto original. Hemos encontrado tres excepciones en las que el texto meta en euskera mantiene, en mayor o menor medida, elementos de la portada original. Veamos dos de ellas a modo de ejemplo:

En la figura 6 se puede ver que en las dos portadas está presente la mano que sujeta la pluma. Aquí se trata de una traducción directa. La portada de la versión en euskera de la figura 7 , sin embargo, es casi idéntica a la original, y en esta ocasión nos encontramos con una traducción indirecta.
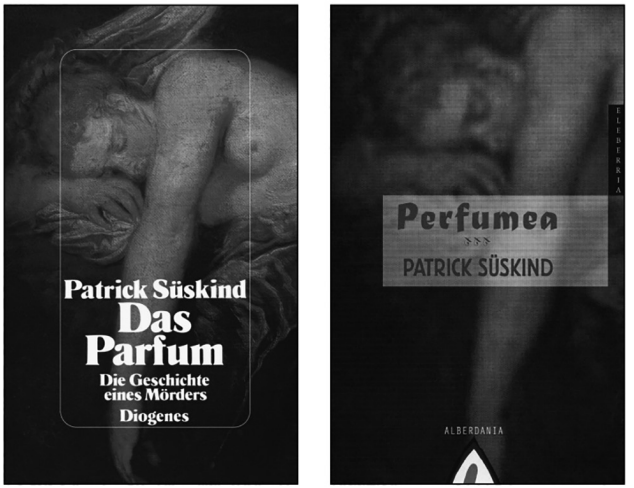

Figura 7. Portadas de Das Parfum (Süskind, 1985) y Perfumea (Süskind, 2007)

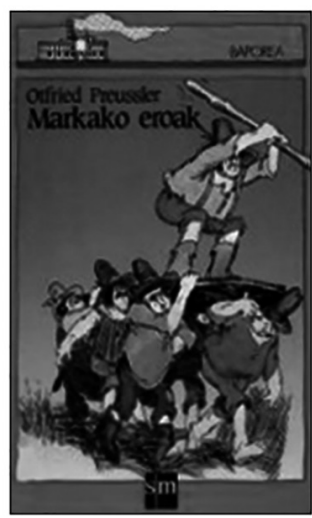

Figura 8. Portadas de Bei uns in Schilda (Preußler, 1958), Los locos de Villasimplona (Preußler, I988) y Markako eroak (Preußler, 1987)

En los textos de LIJ, por otro lado, la tendencia es la contraria; es decir, en I8 libros de 24 se ha mantenido la portada del original, aunque no siempre la del original alemán, sino a veces la de la versión intermedia. Son 7 las veces en las que se ha optado por utilizar la misma portada que la de la versión intermedia en español, a pesar de que dos ellas se han traducido directamente del alemán. Vemos un ejemplo en la figura 8.

Entre los 2 restantes que han mantenido la portada del original alemán, cuatro son traducciones indirectas. 


\section{CONCLUSIONES}

Gracias a los datos obtenidos del catálogo Aleuska y de la descripción del subcatálogo LA-LIJ —como, el número de traducciones, la distribución de los textos por género, la evolución del número de publicaciones por año, el modo de traducción-, se han podido establecer los criterios para la compilación del corpus. Para el proceso de creación de corpus, además, disponer de resultados preliminares acerca del modo de traducción nos ha permitido incluir la versión intermedia cuando las traducciones se han realizado de forma indirecta. Así, y gracias a la herramienta de creación de corpus TAligner, que permite alinear múltiples textos de forma simultánea, se ha podido crear un corpus digitalizado, paralelo y multilingüe.

El análisis paratextual, en un segundo nivel, ha servido para concluir, entre otros, la falta de visibilidad del proceso traductor mediante la información paratextual de los textos meta; es decir, resulta difícil identificar el original de una traducción atendiendo a los datos que ofrecen los paratextos. En este sentido, ha sido de gran ayuda la información epitextual, la obtenida gracias a traductores, editoriales $u$ otras fuentes de internet. Cuando hemos analizado la traducción de dichos elementos paratextuales, hemos podido observar que en ciertas ocasiones (en la traducción de los capítulos) hay indicios que apuntan al uso de versiones intermedias en traducciones directas, pero en otros casos (la traducción de títulos y portadas), resulta más difícil establecer la relación del TO con el TM, porque puede haber varios agentes que han podido influir en el trasvase de un idioma a otro de títulos y portadas. Sin duda, el análisis microtextual posterior podrá ayudar a afinar los resultados obtenidos en los niveles anteriores.

Además, de los resultados de estos análisis se puede extraer otra conclusión clara: la dicoto- mía traducción directa/indirecta no es suficiente para describir la relación entre los textos. En el análisis paratextual y macrotextual hemos podido observar que en las traducciones directas a veces se utilizan otras versiones en mayor o menor medida. Estas, por ejemplo, se podrían describir como traducciones parcialmente directas o traducciones directas compiladas. En las traducciones indirectas, por otro lado, ha habido ocasiones en que los traductores se han valido de hasta tres versiones para realizar la traducción, por lo que se podría hablar de traducciones indirectas compiladas. En resumen, el análisis muestra que hay una amplia gama de posibilidades en el establecimiento de la relación de los TO y los TM, que en nuestro caso ha sido necesario describir y tener en cuenta durante todo el proceso de investigación.

RECIBIDO EN ENERO DE $20 I 7$

ACEPTADO EN AGOSTO DE 2018

VERSIÓN FINAL DE SEPTIEMBRE DE 2018

\section{BIBLIOGRAFÍA}

Barambones, Josu (2009): La traducción audiovisual en ETB-I: Estudio descriptivo de la programación infantil y juvenil, Bilbao: Universidad del País Vasco.

Biguri, Koldo y Pello Zabaleta (i99o): «Pello Zabaletarekin solasean», Senez, ro.

Brezina, Thomas (1995): Grüße aus dem Geisterschloss, München: Bertelsmann.

Ende, Michael (1982): Das Gauklermärchen, Stuttgart: Thienemann.

- (1986): Jojo: historia de un saltimbanqui, trad. Flora Casas y Analis Gruber, Madrid: Debate.

- (1995): Jojo, pailazo baten historia, trad. Xabier Mendiguren, Bilbao: Desclée de Brouwer.

Fischer, Martin B. (20II): «Hexe Lilli-Kika Superbruja», Anuario de investigación en literatura infantil y juvenil, 9, 71-86. 
Garrido Vilariño, Xoán Manuel (2OII): «The paratranslation of the works of Primo Levi», en Federico M. Federici (ed.), Translating Dialects and Languages of Minorities. Challenges and Solutions, Bern: Peter Lang.

Genette, Gérard (1987): Seuil, Paris: Éditions du Seuil.

Gerber, Leah (2008): «Paratextual mediation in translation: Translating the titles of Australian children's fiction into German, 1945-the present», Monash University Linguistics Papers, 6, 4I-50.

Grass, Günter (1979): Das Treffen in Telgte, Darmstadt: Luchterhand.

- (2002): Batzarra Telgten, trad. Andolin Eguzkitza, Irún: Alberdania.

Gutiérrez Lanza, Camino (2007): «Traducción inglés-español y censura de textos cinematográficos: definición, construcción y análisis del corpus o/catálogo TRACEci (I95I-I98I)», en Raquel Merino (ed.), Traducción y censura en España (1939-I985). Estudios sobre corpus TRACE: cine, narrativa, teatro, UPV/EHU: Servicio Editorial de la Universidad del País Vasco.

Hermans, Theo (1999): Translation in Systems. Descriptive and system-oriented approaches explained, Manchester: St. Jerome.

Kästner, Erich (I949): Das doppelte Löttchen, Zürich: Atrium.

- (1957): Las dos Carlotas, trad. Pilar Guerra, Barcelona: Juventud.

- (1992): Lotte eta Luisa, hau bizki parea!, trad. Antton Narbaiza, Donostia: Elkar.

Lambert, José y Hendrik van Gorp (I985): «On describing translations», en Theo Hermans (ed.), The manipulation of literature. Studies in literary translation, Sydney: Croom Helm.

López Gaseni, José Manuel (2008): «Literatura traducida», Portal de la literatura vasca, <http://www. basqueliterature.com/basque/historia/itzulia > [consulta: 27-I-20I7].

Manterola, Elizabete (20II): Euskal literatura beste bizkuntza batzuetara itzulia. Bernardo Atxagaren lanen itzulpen moten arteko alderaketa, Bilbao: Universidad del País Vasco.

Massanek, Joachim (2003): Juli, die Viererkette, Frankfurt am Main: Baumhaus Verlag.

- (2005): Juli, el mejor defensa, trad. Rosa M. Sala Carbó, Barcelona: Planeta.

- (2010): Juli, defentsa onena, trad. Nuria Sebrango, Bilbao: Gero.
Merino, Raquel (I994): Traducción, tradición y manipulación: teatro inglés en España 1950-1990, León: Universidad de León; Bilbao: Universidad del País Vasco.

- (2003): «TRAducciones Censuradas inglésespañol: del catálogo al corpus Trace (teatro)», en Ricardo Muñoz (ed.), Actas del I Congreso Internacional de la Asociación Ibérica de Estudios de Traducción e Interpretación, Granada: Universidad de Granada.

Nöstlinger, Christine (I982): Das Austauschkind, Weinheim: Beltz \& Gelberg.

- (I986): Intercambio con un inglés, trad. Luis Pastor, Madrid: Espasa-Calpe.

- (I99I): Ingeles bat etxean, trad. Xabier Mendiguren, Lizarra: Elkar.

Preussler, Otfried (1958): Bei uns in Schilda, Stuttgart: Thienemann.

- (I987): Markako eroak, trad. Pello Zabaleta, Madrid: SM.

- (I988): Los locos de Villasimplona, trad. Marinella Terzi, Madrid: SM.

RuzickA, Veljka (2002): Kulturelle Regionalisierung in Spanien und literarische Übersetzung. Studien zur Rezeption deutschsprachiger Kinder-und Jugendliteratur in den zweisprachigen autonomen Regionen Baskenland, Galicien und Katalonien, Frankfurt am Main: Peter Lang.

SAnZ-Villar, Zuriñe (20I6): "German-into-Basque/Spanish translation analysis of binomials in a parallel and multilingual corpus», en Gloria Corpas Pastor (ed.), Computerised and Corpus-based Approaches to Phraseology: Monolingual and Multilingual Perspectives, Geneva: Editions Tradulex.

- (20I8): «Interference and the translation of phraseological units in a parallel and multilingual corpus», Meta, I, vol. 63, 72-93.

Süskind, Patrick (1985): Das Parfum: die Geschichte eines Mörders, Zürich: Diogenes.

- (2007): Perfumea: biltzaile baten historia, trad. Miren Arratibel, Irún: Alberdania.

TAHIR-GÜrÇAĞLAR, Şehnaz (2002): «What texts don't tell: The use of paratexts in translation research», en The Hermans (ed.), Crosscultural Transgressions, Manchester: St. Jerome.

Torrealdai, Joan Mari (2013): «Euskal Liburugintza 20I2", Jakin, I98, II-52.

Toury, Gideon (2012): Descriptive Translation Studies and Beyond, Amsterdam: John Benjamins. 
URIBARRI, Ibon (2005): «Deutsch-Baskische Übersetzung: Allgemeine Situation und spezifische Probleme», en Macià Riutort y Jordi Janè (eds.), Deutsch-spanische Zwischenwelten - Neue Horizon148 te für die spanische Germanistik zu Beginn des 21. Jahrhunderts. Tarragona: Forum, <http://usuaris.
tinet.cat/asgc/Forum/Autors/uribarri/uribarri2. html> [consulta: 27-I-20I7].

Zubillaga, Naroa (2013): Alemanetik euskaratutako haur eta gazte literatura: zuzeneko nabiz zeharkako itzulpenen azterketa corpus baten bidez, Bilbao: Universidad del País Vasco. 